\title{
Novel anticancer activity and anticancer mechanisms of Brassica oleracea L. var. capitata f. rubra.
}

\begin{abstract}
Aim of the study: The anticancer activity of red cabbage (RC) and the underlying mechanisms against human cervical and hepatocarcinoma cancer cells were explored in this study. Materials and methods: The cytotoxic activity of RC extract was assessed using 3-(4,5dimethylthiazol-2-yl)-5-(3-carboxymethoxyphenyl)-2-(4-sulfophenyl)-2H-tetrazolium (MTS) cytotoxicity assay. ELISA was used to detect the level of anticancer cytokines, TNF $\alpha$ and IFN $\beta$, in the culture supernatants of cancer cells. Apoptotic cells were investigated by flow cytometry. The levels of apoptotic genes (Bax, BCL-2, Capsases 7-9), cell cycle regulatory genes (cyclin D, E, and A) and tumor suppressor proteins (p27, p21, and p53) were assessed by real time RT-PCR. Results: The cytotoxic effect of the extract on normal human cells was significantly different from its effects on HeLa and HepG2 cells, $251.28 \pm 4.3,23.38 \pm 1.87$, and $28.66 \pm 2.85 \mathrm{mg} / \mathrm{ml}$, respectively. The selectivity index (SI) was $10.88 \pm 0.82$ for HeLa and $8.93 \pm 0.81$ for HepG2 cells. Increased levels of TNF $\alpha$, but not IFN $\beta$ were observed in the treated HeLa and HepG2 culture supernatants when compared with untreated cells. RC extract induced G0/G1 phase arrest in the cancer cells. The extract induced apoptosis, in a dose and time dependent manner, in treated HeLa and HepG2 cells while no observed apoptosis was found in untreated cells. Moreover, RC IC50 showed remarkable influence on the expression of the apoptosis-related genes in a positive and negative manner on both HeLa and HepG2 cells. Discussion/conclusion: RC extract could be considered as a novel anticancer agent providing new prospects for anticancer therapy using a natural product.
\end{abstract}

Keyword: Brassica oleracea; Anticancer; Apoptosis; Cell cycle arrest; Anticancer cytokines 